\title{
CORRELAÇÕES ENTRE CARACTERES DA PLANTA E DO CACHO EM BANANEIRA (Musa spp) ${ }^{1}$
}

\author{
Correlations among characters of the plant and of the bunch in banana (Musa spp.)
}

\author{
Sérgio Luiz Rodrigues Donato ${ }^{2}$, Sebastião de Oliveira e Silva ${ }^{3}$, Orlando Antônio Lucca Filho ${ }^{4}$, \\ Marcelo Bezerra Lima ${ }^{5}$, Herbat Domingues ${ }^{6}$, Juliana da Silva Alves ${ }^{7}$
}

\begin{abstract}
RESUMO
Os caracteres observados em áreas experimentais têm natureza fenotípica e suas correlações são estimadas visando mensurar alterações em um caráter quando se altera outro relacionado. Objetivou-se com este trabalho, quantificar a relação entre os caracteres da planta e do cacho observados na época do florescimento e da colheita de 13 genótipos (variedades e híbridos) de bananeira (Musa spp.), em dois ciclos de produção, em Guanambi, Bahia. Foram utilizadas as variedades Prata anã, Pacovan (AAB), Grande naine e Nanicão (AAA), e os híbridos PA42-44, PV42-85, PV42-142, PV42-68 e ST12-31 (AAAB) e Ambrosia, Calipso, Bucaneiro e FHIA-02 (AAAA), selecionados na Embrapa Mandioca e Fruticultura. Consideraram-se os caracteres: altura da planta; perímetro do pseudocaule; número de folhas vivas no florescimento e na colheita; número de dias do plantio ao florescimento e à colheita; intervalo florescimento à colheita; peso do cacho, da ráquis e da pencas; comprimento e diâmetro do engaço; número de pencas e de frutos; peso da segunda penca; peso, comprimento e diâmetro do fruto e espessura da casca. As correlações entre o peso do cacho e os demais caracteres estudados, variaram entre genótipos e ciclos. As associações entre o peso do cacho e os caracteres da planta, de forma geral foram não-significativas, e entre o peso do cacho e os caracteres do cacho significativas e positivas para a maioria dos genótipos, nos ciclos avaliados. As correlações entre os caracteres envolvendo todos os genótipos ao longo dos dois ciclos foram predominantemente positivas e não- significativas, entretanto, as associações entre os caracteres do cacho foram em maioria significativas, positivas e com valores expressivos.
\end{abstract}

Termos para indexação: Híbridos, variedades, produção, descritores fenotípicos, ciclos, Musa.

\begin{abstract}
The characters observed in a experimental areas has phenotypic nature and their correlations are estimated with the purpose to verify alterations in one character when is altered another. The objectives of this work were to quantify the relationships among characters development and yield attributes in flowering and harvesting periods of 13 banana (Musa spp.) genotypes (varieties and hybrids), in Guanambi State of Bahia, Brazil. The varieties were Prata anã e Pacovan (AAB), Grande naine e Nanicão (AAA), and the hybrids PA42-44, PV42-85, PV42-142, PV42-68 e ST12-31 (AAAB) e Ambrosia, Calipso, Bucaneiro e FHIA-02 (AAAA), selected in Embrapa Mandioca e Fruticultura. The characters analyzed were plant height; pseudostem perimeter; number of functional leaves in the flowering and in the harvesting; number of days from planting to flowering and to harvesting; number of days from flowering to harvesting; bunch weight, of raquis and of the hands; length and diameter of the peduncle; number of hands and fruits; weight of the second hand; weight, length and diameter of finger and peel thickness. The correlations among the weight of the bunch and the other studied characters, varied among the genotypes and cycles. The associations among the weight of the bunch and the characters of the plant, in a general were no significant, and among the weight of the bunch and the characters of the bunch significant and positive for most of the genotypes in the two cycles evaluated. The correlations among the characters involving all the genotypes along the two cycles was predominantly positive and no significant, however, the associations among the characters of the bunch were in majority significant, positive and with expressive values.
\end{abstract}

Index terms: Hybrids, varieties, production, phenotipcs descriptors, cycles, Musa.

\section{(Recebido para publicação em 27 de janeiro de 2005 e aprovado em 19 de setembro de 2005)}

\section{INTRODUÇÃO}

Um dos entraves relevantes à bananicultura brasileira é a falta de variedades comerciais produtivas com porte adequado, resistentes às principais pragas e doenças e adaptadas a diferentes ecossistemas. Constitui estratégia para a solução deste problema o desenvolvimento de cultivares que atendam os requisitos supra citados, mediante programas de melhoramento genético, bem como, sua avaliação e caracterização em áreas de produção quando são comparadas às cultivares tradicionais (SILVA et al., 2000, 2002).

\footnotetext{
${ }^{1}$ Parte da Dissertação do primeiro autor, apresentada à Universidade Federal de Pelotas, para obtenção do Título de Mestre.

2 Engenheiro Agrônomo, Mestre, Professor EAFAJT - Cx. P. 009 - 46.430-000 - Guanambi, BA - sergiodonato@eafajt.gov.br

${ }^{3}$ Engenheiro Agrônomo, Doutor, Pesquisador - Embrapa Mandioca e Fruticultura - Cx. P. 7 - 44.380-000 - Cruz das Almas, BA ssilva@cnpmf.embrapa.br

${ }^{4}$ Engenheiro Agrônomo, Doutor, Professor UFPEL/FAEM - Cx. P. 354 - 96.001-970 - Pelotas, RS - luccafilho@ufpel.tche.br

${ }^{5}$ Engenheiro Agrônomo, Mestre, Pesquisador - Embrapa mandioca e Fruticultura - Cx. P. 7 - 44.380-000 - Cruz das Almas, BA mlima@cnpmf.embrapa.br

${ }^{6}$ Técnico em Agropecuária/EAFAJT - Cx. P. 009 - 46.430-000 - Guanambi, BA.

${ }^{7}$ Estudante, AGRFBA - Bolsista da FAPESB/Embrapa - Cruz das Almas, BA - jsaagr@bol.bom.br
} 
Os caracteres normalmente estudados em trabalhos de avaliação de genótipos são: ciclo da cultura, altura da planta, perímetro do pseudocaule, peso do cacho, número de frutos por cacho, comprimento e diâmetro dos frutos, descritores relevantes para a identificação e a seleção de indivíduos superiores (FLORES, 2000; SILVA et al., 2000).

As correlações determinadas entre caracteres observados nos ensaios experimentais são atribuídas a fatores genéticos e ambientais (VENCOVSKY \& BARRIGA, 1992) e estimadas com o propósito de mensurar a alteração em um caráter quando se altera outro. Ao determinar a magnitude e a significância das associações entre descritores fenotípicos, utilizados para seleção de indivíduos em trabalhos de avaliação, pode-se discriminar quais influenciam na produção.

A correlação, cuja descrição numérica é adimensional, pode assumir valores negativos, quando as variáveis variam em proporção inversa ou positiva, se estas variarem em proporção direta (RIBEIRO JÚNIOR, 2001).

Diversos autores estudaram as correlações entre os descritores usuais nos trabalhos de avaliação de genótipos de bananeira (FERNANDEZ-CALDAS et al., 1977; GARCIA et al., 1977; HASSELO, 1962; HOLDER \& CUMBS, 1982; IUCHI et al., 1979; JARAMILLO, 1982; LIMA NETO et al., 2003; LOSSOIS, 1963; PÁDUA, 1978; SIQUEIRA, 1984; VARGAS, 1983).

Os estudos de Vargas (1983), comparando os clones 'Grande naine' e 'Valery', revelaram a existência de uma correlação linear entre peso do cacho e perímetro do pseudocaule. Outros trabalhos também obtiveram correlações significativas e positivas entre estes descritores (FERNANDEZ-CALDAS et al., 1977; GARCIA et al., 1977; HASSELO, 1962; HOLDER \& CUMBS, 1982; IUCHI et al., 1979; LIMA NETO et al., 2003; LOSSOIS, 1963; SIQUEIRA, 1984). A altura da planta não apresentou correlação significativa com a produção em bananeira 'Prata' (IUCHI et al., 1979; SIQUEIRA, 1984), embora, Lima Neto et al. (2003) tenham encontrado correlações positivas e significativas para estes caracteres na maioria dos genótipos estudados.

O número de frutos produzidos por um genótipo é fundamental na determinação do tamanho e do peso do cacho (CARVALHO, 1995). Este caráter está estreitamente relacionado com o número de pencas, sendo que o acréscimo de uma penca aumenta o número de frutos em 24,6\% (JARAMILLO, 1982). Estes mesmos autores encontraram correlações positivas e significativas entre o peso do cacho e o número de frutos, e entre o comprimento dos frutos e o peso médio dos frutos para a maioria dos genótipos estudados.

Vargas (1983) encontrou uma relação entre comprimento do engaço e tamanho do fruto, afirmando ser uma característica de variação genética. Existe uma correlação bem definida entre diâmetro do fruto do dedo central da segunda penca e peso do cacho (JARAMILLO, 1982). Estimativas positivas e altas para as correlações entre o diâmetro dos frutos com o peso das pencas e do cacho, e o comprimento dos frutos com o peso das pencas e do cacho em bananeira 'Prata', foram obtidas por Pádua (1978).

Assim, objetivou-se com este trabalho, foi estimar correlações entre caracteres da planta e do cacho observados na época do florescimento e da colheita de 13 genótipos (variedades e híbridos) de bananeira (Musa spp.), em dois ciclos produtivos, em Guanambi (Sudoeste da Bahia).

\section{MATERIAL E MÉTODOS}

O trabalho foi implantado num LATOSSOLO VERMELHO-AMARELO distrófico na área experimental da Escola Agrotécnica Federal Antônio José Teixeira, em Guanambi, BA.

Foram avaliadas em dois ciclos de produção as variedades tipo Prata: Prata anã e Pacovan (AAB); tipo Cavendish: Grande naine e Nanicão (AAA); os híbridos tipo Prata: PA42-44, PV42-85, PV42-142, PV42-68 e ST12-31 (AAAB), gerados e selecionados pela Embrapa Mandioca e Fruticultura; o tipo Gros Michel: Ambrosia, Calipso e Bucaneiro (AAAA), e o híbrido tipo Cavendish FHIA-02 (AAAA) (Tabela 1), introduzidos e selecionados pela Embrapa, da Jamaica e de Honduras, respectivamente.

Foram avaliados caracteres observados nos estádios de florescimento e colheita do cacho de cada planta útil: altura da planta (APL), expressa em metros; perímetro do pseudocaule (PPS), em centímetros; número de dias do plantio ao florescimento (NDF); número de folhas vivas no florescimento (NFF); número de dias do plantio à colheita do cacho (NDC); período do florescimento a colheita (PFC); número de folhas vivas na colheita (NFC); peso do cacho (PCA), da ráquis (PRA) e das pencas (PPE), em quilogramas; comprimento do engaço (CEN), em centímetros; diâmetro do engaço (DEN), em milímetros; número de pencas (NPE) e de frutos por cacho (NFR); peso da segunda penca (PSP), em quilogramas; peso médio do fruto (PMF), em gramas, comprimento externo (CEF) e interno (CIF) dos frutos, em centímetros, diâmetro do fruto (DMF), em milímetros, e espessura da casca (ECA), em milímetros, determinados

Ciênc. agrotec., Lavras, v. 30, n. 1, p. 21-30, jan./fev., 2006 
no fruto central da fileira externa de frutos da segunda penca.

O delineamento experimental foi em blocos casualizados com 13 tratamentos, cinco repetições, sendo cada parcela constituída por 20 plantas, com seis centrais úteis, plantadas no espaçamento de 3,0 m x 2,0 m. Utilizouse a variedade Prata anã, como bordadura para o experimento.

Para cada genótipo e cada ciclo de produção, foram estimadas as correlações referentes às associações entre o peso do cacho e os 20 demais caracteres, observados na época do florescimento e da colheita do cacho. Também foram calculadas todas as associações possíveis para os
21 caracteres considerando-se simultaneamente todos os genótipos ao longo dos dois ciclos, com base na correlação de Pearson (GOMES, 1985).

\section{RESULTADOS E DISCUSSÃO}

As correlações entre o caráter peso do cacho e os caracteres observados na época do florescimento, altura da planta, perímetro do pseudocaule, número de folhas vivas no florescimento e número de dias do plantio ao florescimento para cada genótipo de bananeira, e cada ciclo, bem como os respectivos testes de significância, encontram-se na Tabela 2.

TABELA 1 - Algumas características dos genótipos de bananeira avaliados na Escola Agrotécnica Federal Antônio José Teixeira, Guanambi, BA, 2002 a 2003.

\begin{tabular}{|c|c|c|}
\hline Genótipo & $\begin{array}{l}\text { Grupo } \\
\text { Genômico }\end{array}$ & Características \\
\hline Prata anã & $\overline{\mathrm{AAB}}$ & $\begin{array}{l}\text { Variedade tipo Prata, mutante de Branca, porte baixo a médio, de baixa } \\
\text { produtividade, suscetível às sigatokas amarela e negra e ao mal-do-panamá. }\end{array}$ \\
\hline Pacovan & $\mathrm{AAB}$ & $\begin{array}{l}\text { Variedade tipo Prata, mutante da Prata comum, porte alto, de baixa } \\
\text { produtividade, suscetível às sigatokas amarela e negra e ao mal-do-panamá. }\end{array}$ \\
\hline Grande naine & AAA & $\begin{array}{l}\text { Variedade tipo Cavendish, mutante da Nanica, porte médio a baixo, alta } \\
\text { produtividade, suscetível às sigatokas amarela e negra e resistente ao mal-do- } \\
\text { panamá. }\end{array}$ \\
\hline Nanicão & $\mathrm{AAA}$ & $\begin{array}{l}\text { Variedade tipo Cavendish, mutante da Nanica, porte médio, alta produtividade, } \\
\text { suscetível às sigatokas amarela e negra e resistente ao mal-do-panamá. }\end{array}$ \\
\hline PA42-44 & AAAB & $\begin{array}{l}\text { Híbrido tipo Prata, (Prata anã x M53), porte baixo a médio, resistente às } \\
\text { sigatokas amarela e negra. }\end{array}$ \\
\hline PV42-85 & AAAB & $\begin{array}{l}\text { Híbrido tipo Prata (Pacovan x M53), porte alto, resistente às sigatokas amarela, } \\
\text { negra e mal-do-panamá. }\end{array}$ \\
\hline PV42-68 & $\mathrm{AAAB}$ & $\begin{array}{l}\text { Híbrido tipo Prata (Pacovan x M53), porte alto, resistentes às sigatokas amarela, } \\
\text { negra e mal-do-panamá. }\end{array}$ \\
\hline PV42-142 & $\mathrm{AAAB}$ & $\begin{array}{l}\text { Híbrido tipo Prata (Pacovan x M53), porte alto, resistente às sigatokas amarela, } \\
\text { negra e mal-do-panamá. }\end{array}$ \\
\hline ST12-31 & AAAB & $\begin{array}{l}\text { Híbrido tipo Prata (Prata São tomé x Lidi), porte alto, resistente à sigatoka } \\
\text { amarela e mal-do-panamá, suscetível à sigatoka negra. }\end{array}$ \\
\hline Ambrósia & AAAA & $\begin{array}{l}\text { Híbrido tipo Gros Michel, derivado de Highgate, porte médio a alto, resistente } \\
\text { ao mal-do-panamá e a sigatoka negra. }\end{array}$ \\
\hline Bucaneiro & AAAA & $\begin{array}{l}\text { Híbrido tipo Gros Michel, derivado de Highgate, porte médio a alto, resistente } \\
\text { ao mal-do-panamá e a sigatoka negra. }\end{array}$ \\
\hline Calipso & AAAA & $\begin{array}{l}\text { Híbrido tipo Gros Michel, derivado de Highgate, porte médio a alto, resistente } \\
\text { ao mal-do-panamá e a sigatoka negra. }\end{array}$ \\
\hline FHIA-02 & AAAA & $\begin{array}{l}\text { Híbrido tipo Cavendish (Williams x SH33-93), porte baixo, resistente às } \\
\text { sigatokas negra e amarela, suscetível ao mal-do-panamá, introduzido da FHIA }{ }^{1} \text {. }\end{array}$ \\
\hline
\end{tabular}


TABELA 2 - Correlações fenotípicas entre o peso do cacho e os caracteres observados na época do florescimento para cada genótipo, no primeiro e segundo ciclos de produção, com os respectivos testes de significância, Guanambi, BA, 2002 a 2003.

\begin{tabular}{|c|c|c|c|c|c|}
\hline \multirow[t]{2}{*}{ Genótipos } & \multicolumn{5}{|c|}{ Correlação entre o peso do cacho e os caracteres 1} \\
\hline & Ciclo & APL & PPS & NFF & NDF \\
\hline \multirow[t]{2}{*}{ ST12-31 } & $1^{\circ}$ & $0,25^{\text {ns }}$ & $0,35^{\text {ns }}$ & $0,72^{\text {ns }}$ & $-0,92^{*}$ \\
\hline & $2^{\circ}$ & $0,97^{*}$ & $0,80^{*}$ & $0,75^{\text {ns }}$ & $-0,97^{*}$ \\
\hline \multirow[t]{2}{*}{ PV42-68 } & $1^{\circ}$ & $0,36^{\mathrm{ns}}$ & $0,02^{\text {ns }}$ & $-0,07^{\mathrm{ns}}$ & $-0,61^{\mathrm{ns}}$ \\
\hline & $2^{\circ}$ & $-0,85^{*}$ & $-0,55^{\mathrm{ns}}$ & $0,85^{*}$ & $-0,51^{\mathrm{ns}}$ \\
\hline \multirow[t]{2}{*}{ PV42-142 } & $1^{\circ}$ & $-0,88^{*}$ & $0,03^{\text {ns }}$ & $0,95^{*}$ & $-0,95^{*}$ \\
\hline & $2^{\circ}$ & $-0,90^{*}$ & $-0,55^{\mathrm{ns}}$ & $-0,16^{\mathrm{ns}}$ & $-0,63^{\mathrm{ns}}$ \\
\hline \multirow[t]{2}{*}{ Pacovan } & $1^{\circ}$ & $0,65^{\mathrm{ns}}$ & $0,24^{\mathrm{ns}}$ & $0,59^{\text {ns }}$ & $-0,09^{\mathrm{ns}}$ \\
\hline & $2^{\circ}$ & $-0,16^{\mathrm{ns}}$ & $0,25^{\mathrm{ns}}$ & $0,76^{\mathrm{ns}}$ & $-0,10^{\mathrm{ns}}$ \\
\hline \multirow[t]{2}{*}{ PV42-85 } & $1^{\circ}$ & $-0,09^{\mathrm{ns}}$ & $0,93^{*}$ & $0,84^{*}$ & $-0,86^{*}$ \\
\hline & $2^{\circ}$ & $0,10^{\mathrm{ns}}$ & $0,03^{\text {ns }}$ & $-0,00^{\mathrm{ns}}$ & $-0,21^{\mathrm{ns}}$ \\
\hline \multirow[t]{2}{*}{ Ambrosia } & $1^{\circ}$ & $-0,06^{\mathrm{ns}}$ & $0,44^{\mathrm{ns}}$ & $0,03^{\mathrm{ns}}$ & $-0,55^{\mathrm{ns}}$ \\
\hline & $2^{\circ}$ & $0,52^{\text {ns }}$ & $0,37^{\text {ns }}$ & $0,25^{\mathrm{ns}}$ & $-0,21^{\mathrm{ns}}$ \\
\hline \multirow[t]{2}{*}{ Calipso } & $1^{\circ}$ & $0,80^{*}$ & $-0,67^{\mathrm{ns}}$ & $0,78^{*}$ & $-0,47^{\mathrm{ns}}$ \\
\hline & $2^{\circ}$ & $0,31^{\mathrm{ns}}$ & $0,64^{\mathrm{ns}}$ & $-0,10^{\mathrm{ns}}$ & $0,02^{\mathrm{ns}}$ \\
\hline \multirow{2}{*}{ Bucaneiro } & $1^{\circ}$ & $0,27^{\mathrm{ns}}$ & $0,73^{\text {ns }}$ & $-0,22^{\mathrm{ns}}$ & $0,32^{\mathrm{ns}}$ \\
\hline & $2^{\circ}$ & $0,48^{\text {ns }}$ & $0,65^{\text {ns }}$ & $0,39^{\mathrm{ns}}$ & $-0,91^{*}$ \\
\hline \multirow[t]{2}{*}{ Nanicão } & $1^{\circ}$ & $0,76^{\text {ns }}$ & $0,87^{*}$ & $-0,16^{\mathrm{ns}}$ & $-0,93^{*}$ \\
\hline & $2^{\circ}$ & $0,69^{\text {ns }}$ & $0,85^{*}$ & $0,20^{\mathrm{ns}}$ & $-0,62^{\mathrm{ns}}$ \\
\hline \multirow[t]{2}{*}{ PA42-44 } & $1^{\mathrm{o}}$ & $0,68^{\mathrm{ns}}$ & $0,08^{\mathrm{ns}}$ & $0,89^{*}$ & $0,25^{\mathrm{ns}}$ \\
\hline & $2^{\circ}$ & $0,40^{\mathrm{ns}}$ & $-0,10^{\mathrm{ns}}$ & $-0,41^{\mathrm{ns}}$ & $-0,74^{\mathrm{ns}}$ \\
\hline \multirow[t]{2}{*}{ Grande naine } & $1^{\circ}$ & $-0,08^{\mathrm{ns}}$ & $0,28^{\mathrm{ns}}$ & $0,04^{\mathrm{ns}}$ & $-0,75^{\mathrm{ns}}$ \\
\hline & $2^{\circ}$ & $0,31^{\mathrm{ns}}$ & $0,84^{*}$ & $0,89^{*}$ & $-0,92^{*}$ \\
\hline \multirow[t]{2}{*}{ Prata anã } & $1^{\circ}$ & $0,77^{\mathrm{ns}}$ & $0,58^{\mathrm{ns}}$ & $0,09^{\mathrm{ns}}$ & $0,89^{*}$ \\
\hline & $2^{\circ}$ & $0,61^{\mathrm{ns}}$ & $0,59^{\mathrm{ns}}$ & $0,56^{\mathrm{ns}}$ & $0,65^{\mathrm{ns}}$ \\
\hline \multirow[t]{2}{*}{ FHIA-02 } & $1^{\mathrm{o}}$ & $-0,73^{\mathrm{ns}}$ & $-0,14^{\mathrm{ns}}$ & $0,46^{\mathrm{ns}}$ & $-0,95^{*}$ \\
\hline & $2^{\circ}$ & $-0,55^{\mathrm{ns}}$ & $0,48^{\text {ns }}$ & $-0,27^{\mathrm{ns}}$ & $-0,70^{\mathrm{ns}}$ \\
\hline
\end{tabular}

${ }^{1 / A P L}$ : Altura da planta; PPS: Perímetro do pseudocaule; NFF: Número de folhas vivas no florescimento; NDF: Número de dias do plantio ao florescimento.

*:Significativo ao nível de $5 \%$ de probabilidade. ns: não-significativo ao nível de $5 \%$ de probabilidade.

As associações entre peso do cacho e altura da planta para a maioria dos genótipos foram não-significativas e positivas nos dois ciclos de produção e os valores oscilaram bastante entre os ciclos, sendo que PV42-142, Nanicão e Prata anã mostraram estabilidade. Esta associação mostrouse significativa e em sua maioria negativa e alta, nos genótipos ST12-31, PV42-68 e Pacovan no segundo ciclo, em Calipso no primeiro ciclo e PV42-142 nos dois ciclos, genótipos estes, de porte alto, o que significa que o peso do cacho variou para os mesmos, de forma inversa com o porte da planta, o que corrobora as afirmações de Donato (2003) de que, independente do grupo genômico, os genótipos de porte mais baixo foram mais produtivos que os de porte mais alto. Este comportamento pode ter sido induzido por condições ambientais, uma vez que ocorreram velocidades do vento críticas para a cultura, durante o ciclo de produção, o que pode ter afetado a produtividade de determinados genótipos, pois a influência deletéria do vento é maior nas cultivares de porte mais alto.

De forma semelhante, as estimativas entre peso do cacho e perímetro do pseudocaule foram predominantemente não-significativas e positivas nos dois ciclos de produção. As estimativas significativas encontradas foram positivas, com valores elevados e ocorreram nos genótipos ST12-31 e Grande naine (segundo ciclo), PV42-85 (primeiro ciclo) e Nanicão (primeiro e segundo ciclos). As estimativas

Ciênc. agrotec., Lavras, v. 30, n. 1, p. 21-30, jan./fev., 2006 
oscilaram bastante nos ciclos, constituindo exceção os genótipos Pacovan, Ambrosia, Bucaneiro, Nanicão e Prata anã. Lima Neto et al. (2003) encontraram correlações significativas e positivas em Grande naine.

A associação peso do cacho e número de folhas no florescimento mostrou estimativas predominantemente nãosignificativas e positivas. Estimativas estatisticamente significativas e positivas foram verificadas em PV42-68 e Grande naine (segundo ciclo), PV42-142, PV42-85, Calipso e PA42-44 (primeiro ciclo). Apenas o híbrido ST12-31 apresentou estabilidade.

A associação entre peso do cacho e número de dias do plantio ao florescimento revelou um maior número de casos significativos, sendo todos negativos e com valores de alta magnitude como nos genótipos ST12-31 (primeiro e segundo ciclos), PV42-142, PV2-85, Bucaneiro, Nanicão, Prata anã e FHIA-02 (primeiro ciclo) e Grande naine (segundo ciclo). As estimativas encontradas foram predominantemente negativas. Os genótipos ST12-31, PV42-68, Pacovan e Nanicão, nesta associação, mostraram relativa estabilidade nos dois ciclos.

As correlações entre o caráter peso do cacho e os caracteres observados na época da colheita, número de dias do plantio à colheita do cacho, intervalo florescimentocolheita, número de folhas vivas na colheita, peso da ráquis e das pencas, comprimento e diâmetro do engaço, número de pencas e de frutos por cacho, para cada genótipo de bananeira, nos dois ciclos de produção, com os respectivos testes de significância, encontram-se na Tabela 3.

TABELA 3 - Correlações fenotípicas entre o peso do cacho e os demais caracteres observados na época da colheita para cada genótipo, no primeiro e segundo ciclos de produção, com os respectivos testes de significância, Guanambi, BA, 2002 a 2003.

\begin{tabular}{|c|c|c|c|c|c|c|c|c|c|c|}
\hline \multirow[t]{2}{*}{ Genótipos } & \multicolumn{10}{|c|}{ Correlação entre o peso do cacho e os caracteres $^{1}$} \\
\hline & Ciclos & PRA & PPE & CEN & DEN & NPE & NFR & NFC & NDC & PFC \\
\hline \multirow[t]{2}{*}{ Grande naine } & $1^{\circ}$ & $0,93^{*}$ & $0,99^{*}$ & $0,19^{\mathrm{ns}}$ & $-0,85^{*}$ & $0,55^{\mathrm{ns}}$ & $0,75^{\mathrm{ns}}$ & $0,01^{\mathrm{ns}}$ & $-0,72^{\mathrm{ns}}$ & $0,84^{*}$ \\
\hline & $2^{\circ}$ & $0,79^{*}$ & $0,99^{*}$ & $0,39^{\mathrm{ns}}$ & $0,91^{*}$ & $0,78^{*}$ & $0,60^{\mathrm{ns}}$ & $0,91^{*}$ & $-0,93^{*}$ & $0,87^{*}$ \\
\hline \multirow[t]{2}{*}{ Nanicão } & $1^{\circ}$ & $0,93^{*}$ & $0,99^{*}$ & $0,64^{\mathrm{ns}}$ & $0,97^{*}$ & $0,99^{*}$ & $0,94^{*}$ & $-0,27^{\mathrm{ns}}$ & $-0,58^{\mathrm{ns}}$ & $0,43^{\mathrm{ns}}$ \\
\hline & $2^{\circ}$ & $0,80^{*}$ & $0,99^{*}$ & $-0,02^{\mathrm{ns}}$ & $0,94^{*}$ & $0,80^{*}$ & $0,92^{*}$ & $0,51^{\mathrm{ns}}$ & $-0,63^{\mathrm{ns}}$ & $0,05^{\mathrm{ns}}$ \\
\hline \multirow[t]{2}{*}{ FHIA-02 } & $1^{\mathrm{o}}$ & $0,96^{*}$ & $0,99^{*}$ & $0,85^{*}$ & $0,70^{\mathrm{ns}}$ & $0,75^{\mathrm{ns}}$ & $0,95^{*}$ & $0,53^{\mathrm{ns}}$ & $-0,46^{\mathrm{ns}}$ & $0,31^{\mathrm{ns}}$ \\
\hline & $2^{\circ}$ & $0,20^{\mathrm{ns}}$ & $0,88^{*}$ & $0,78^{*}$ & $0,25^{\mathrm{ns}}$ & $0,65^{\mathrm{ns}}$ & $0,80^{*}$ & $0,57^{\mathrm{ns}}$ & $-0,35^{\mathrm{ns}}$ & $0,49^{\mathrm{ns}}$ \\
\hline \multirow[t]{2}{*}{ Bucaneiro } & $1^{o}$ & $0,99^{*}$ & $0,97^{*}$ & $0,73^{\mathrm{ns}}$ & $0,91^{*}$ & $0,87^{*}$ & $0,82^{*}$ & $0,65^{\mathrm{ns}}$ & $-0,89^{*}$ & $-0,75^{\mathrm{ns}}$ \\
\hline & $2^{\circ}$ & $0,96^{*}$ & $0,99^{*}$ & $0,91^{*}$ & $0,52^{\mathrm{ns}}$ & $0,95^{*}$ & $0,73^{\mathrm{ns}}$ & $0,82^{*}$ & $-0,64^{\mathrm{ns}}$ & $0,50^{*}$ \\
\hline \multirow[t]{2}{*}{ Calipso } & $1^{\circ}$ & $0,19^{\text {ns }}$ & $0,99^{*}$ & $0,77^{\mathrm{ns}}$ & $0,80^{*}$ & $-0,47^{\mathrm{ns}}$ & $-0,30^{\mathrm{ns}}$ & $0,95^{*}$ & $-0,70^{\mathrm{ns}}$ & $-0,17^{\mathrm{ns}}$ \\
\hline & $2^{\circ}$ & $0,70^{\mathrm{ns}}$ & $0,99^{*}$ & $-0,62^{\mathrm{ns}}$ & $0,76^{\mathrm{ns}}$ & $0,60^{\mathrm{ns}}$ & $0,59^{\mathrm{ns}}$ & $0,43^{\mathrm{ns}}$ & $0,06^{\mathrm{ns}}$ & $0,11^{\mathrm{ns}}$ \\
\hline \multirow[t]{2}{*}{ Ambrosia } & $1^{\circ}$ & $0,40^{\mathrm{ns}}$ & $0,97^{*}$ & $-0,46^{\mathrm{ns}}$ & $0,85^{*}$ & $0,81^{*}$ & $0,56^{\mathrm{ns}}$ & $-0,26^{\mathrm{ns}}$ & $-0,47^{\mathrm{ns}}$ & $0,56^{\mathrm{ns}}$ \\
\hline & $2^{\circ}$ & $0,78^{*}$ & $0,99^{*}$ & $0,36^{\mathrm{ns}}$ & $0,57^{\mathrm{ns}}$ & $0,53^{\mathrm{ns}}$ & $0,38^{\mathrm{ns}}$ & $0,31^{\mathrm{ns}}$ & $-0,30^{\mathrm{ns}}$ & $-0,11^{\mathrm{ns}}$ \\
\hline \multirow[t]{2}{*}{ Prata anã } & $1^{\circ}$ & $0,67^{\mathrm{ns}}$ & $0,98^{*}$ & $0,76^{\mathrm{ns}}$ & $0,91^{*}$ & $0,94^{*}$ & $0,73^{\mathrm{ns}}$ & $0,00^{\mathrm{ns}}$ & $0,39^{\mathrm{ns}}$ & $-0,61^{\mathrm{ns}}$ \\
\hline & $2^{\circ}$ & $0,37^{\mathrm{ns}}$ & $0,99^{*}$ & $0,04^{\mathrm{ns}}$ & $0,16^{\mathrm{ns}}$ & $0,12^{\mathrm{ns}}$ & $0,76^{\mathrm{ns}}$ & $0,40^{\mathrm{ns}}$ & $0,50^{\mathrm{ns}}$ & $-0,65^{\mathrm{ns}}$ \\
\hline \multirow[t]{2}{*}{ PA42-44 } & $1^{\mathrm{o}}$ & $-0,69^{\mathrm{ns}}$ & $0,99^{*}$ & $-0,58^{\mathrm{ns}}$ & $-0,44^{\mathrm{ns}}$ & $0,92^{*}$ & $0,77^{*}$ & $0,60^{\mathrm{ns}}$ & $0,21^{\mathrm{ns}}$ & $0,01^{\mathrm{ns}}$ \\
\hline & $2^{\circ}$ & $0,58^{\mathrm{ns}}$ & $0,92^{*}$ & $0,95^{*}$ & $0,68^{\mathrm{ns}}$ & $0,10^{\mathrm{ns}}$ & $0,58^{\mathrm{ns}}$ & $0,55^{\mathrm{ns}}$ & $-0,85^{*}$ & $-0,62^{\mathrm{ns}}$ \\
\hline \multirow[t]{2}{*}{ Pacovan } & $1^{\circ}$ & $0,78^{*}$ & $0,99^{*}$ & $-0,63^{\mathrm{ns}}$ & $0,95^{*}$ & $0,63^{\mathrm{ns}}$ & $0,44^{\mathrm{ns}}$ & $0,67^{\mathrm{ns}}$ & $0,06^{\mathrm{ns}}$ & $0,21^{\mathrm{ns}}$ \\
\hline & $2^{o}$ & $0,43^{\mathrm{ns}}$ & $0,96^{*}$ & $0,22^{\text {ns }}$ & $0,90^{*}$ & $-0,24^{\mathrm{ns}}$ & $0,22^{\mathrm{ns}}$ & $0,74^{\mathrm{ns}}$ & $-0,17^{\mathrm{ns}}$ & $0,17^{\mathrm{ns}}$ \\
\hline \multirow[t]{2}{*}{ PV42-68 } & $1^{\circ}$ & $0,86^{*}$ & $0,99^{*}$ & $-0,12^{\mathrm{ns}}$ & $0,91^{*}$ & $0,24^{\mathrm{ns}}$ & $0,52^{\mathrm{ns}}$ & $-0,67^{\mathrm{ns}}$ & $-0,95^{*}$ & $-0,16^{\mathrm{ns}}$ \\
\hline & $2^{\circ}$ & $0,72^{\text {ns }}$ & $0,99^{*}$ & $-0,39^{\mathrm{ns}}$ & $0,61^{\mathrm{ns}}$ & $0,93^{*}$ & $0,94^{*}$ & $0,92^{*}$ & $-0,54^{\mathrm{ns}}$ & $-0,60^{\mathrm{ns}}$ \\
\hline \multirow[t]{2}{*}{ PV42-85 } & $1^{\mathrm{o}}$ & $0,89^{*}$ & $0,98^{*}$ & $0,34^{\mathrm{ns}}$ & $0,40^{\mathrm{ns}}$ & $-0,89^{*}$ & $-0,59^{\mathrm{ns}}$ & $0,46^{\mathrm{ns}}$ & $-0,78^{*}$ & $0,63^{\mathrm{ns}}$ \\
\hline & $2^{o}$ & $0,07^{\mathrm{ns}}$ & $0,98^{*}$ & $0,26^{\mathrm{ns}}$ & $0,82^{*}$ & $-0,41^{\mathrm{ns}}$ & $-0,31^{\mathrm{ns}}$ & $-0,46^{\mathrm{ns}}$ & $0,35^{\mathrm{ns}}$ & $0,89^{*}$ \\
\hline \multirow[t]{2}{*}{ PV42-142 } & $1^{\circ}$ & $0,77^{\mathrm{ns}}$ & $0,99^{*}$ & $-0,13^{\mathrm{ns}}$ & $-0,35^{\mathrm{ns}}$ & $-0,10^{\mathrm{ns}}$ & $0,18^{\text {ns }}$ & $-0,09^{\mathrm{ns}}$ & $-0,94^{*}$ & $0,82^{*}$ \\
\hline & $2^{\circ}$ & $0,07^{\mathrm{ns}}$ & $0,91^{*}$ & $-0,67^{\mathrm{ns}}$ & $0,77^{\mathrm{ns}}$ & $0,14^{\mathrm{ns}}$ & $0,19^{\text {ns }}$ & $-0,96^{*}$ & $-0,55^{\mathrm{ns}}$ & $0,46^{\mathrm{ns}}$ \\
\hline \multirow[t]{2}{*}{ ST12-31 } & $1^{\mathrm{o}}$ & $0,96^{*}$ & $0,99^{*}$ & $0,52^{\mathrm{ns}}$ & $0,82^{*}$ & $0,40^{\mathrm{ns}}$ & $0,65^{\mathrm{ns}}$ & $-0,81^{*}$ & $-0,93^{*}$ & $-0,52^{\mathrm{ns}}$ \\
\hline & $2^{\circ}$ & $0,90^{*}$ & $0,99^{*}$ & $0,52^{\mathrm{ns}}$ & $0,77^{\mathrm{ns}}$ & $0,90^{*}$ & $0,94^{*}$ & $0,81^{*}$ & $-0,89^{*}$ & $0,46^{*}$ \\
\hline
\end{tabular}

1/PRA: Peso da ráquis; PPE: Peso das pencas; CEN: Comprimento do engaço; DEN: Diâmetro do engaço; NPE: Número de pencas; NFR: Número de frutos; NFC: Número de folhas vivas na colheita; NDC: Número de dias do plantio à colheita; PFC: Período do florescimento à colheita.

*:Significativo ao nível de $5 \%$ de probabilidade; ns: não-significativo ao nível de $5 \%$ de probabilidade. 
A maioria dos valores encontrados para a associação peso do cacho e da ráquis nos dois ciclos de produção, foram estatisticamente significativos, positivos e elevados. Os genótipos Calipso, Prata anã, PA42-44 e PV42-142 apresentaram valores não-significativos nos dois ciclos. Apenas o híbrido PA42-44 no primeiro ciclo de produção exibiu valor não-significativo e negativo, o que denota um padrão geral de variação direta do peso do cacho com o peso da ráquis, o que era esperado, pois, os cachos são compostos de pencas (frutos) e ráquis, e os cachos grandes têm maiores ráquis. Para cachos com o mesmo número de pencas, a participação percentual da ráquis no peso do cacho oscilou entre 6,7 e 7,3\% para cultivares tipo Cavendish (JARAMILLO, 1982). Donato (2003) encontrou uma variação para a participação da ráquis no peso do cacho de $9,6 \%$ para a variedade Grande naine a 14,83\% para o híbrido PV42-142, no qual de forma geral, a maior participação da ráquis no peso do cacho ocorreu nas cultivares com menores pesos de cachos. Os genótipos Nanicão, Bucaneiro, PV42-68 e ST12-31, apresentaram relativa estabilidade nos valores das estimativas com relação aos dois ciclos.

As correlações entre peso do cacho e das pencas, foram estatisticamente significativas, positivas, e com valores elevados para todos os genótipos avaliados nos dois ciclos de produção, como era de se esperar, pois as pencas são o próprio cacho, apenas sem a ráquis.

As associações entre o peso do cacho e o comprimento do engaço, foram na maioria nãosignificativas, embora tenham ocorrido estimativas significativas nos dois ciclos de produção para o genótipo FHIA-02 e apenas no segundo ciclo para Bucaneiro e PA4244. Somente os híbridos FHIA-02, PV42-85 e ST12-31, mostraram estabilidade para a associação peso do cacho e comprimento do engaço ao longo dos ciclos.

Analogamente à correlação entre peso do cacho e da ráquis, a maioria dos genótipos estudados apresentam valores estatisticamente significativos e positivos para a associação peso do cacho e diâmetro do engaço nos dois ciclos de produção. Correlações negativas foram verificadas para Grande naine, PA42-44 e PV42-142 no primeiro ciclo. As estimativas foram estáveis nos dois ciclos para os genótipos Nanicão, Calipso, Pacovan e ST12-31.

Dos genótipos avaliados, o Calipso, o PV42-142, a Pacovan no primeiro ciclo e o PV42-85 (nos dois ciclos de produção) apresentaram valores negativos para a correlação entre o peso do cacho e o número de pencas. Cerca de $42 \%$ dos valores encontrados para essa associação foram significativos, positivos e apresentaram alta magnitude. Dos valores estatisticamente significativos, apenas o híbrido PV42-85 mostrou valor negativo no primeiro ciclo de produção. Esta associação mostrou-se muito instável nos genótipos estudados, exceto para FHIA02 e Bucaneiro.

A associação peso do cacho e número de frutos, mostrou-se significativa e positiva para a maioria dos casos. Estimativas negativas foram observadas para os híbridos Calipso, no primeiro e para PV42-85 nos dois ciclos de produção. Valores estatisticamente significativos, positivos e de média à alta magnitude, foram verificados para os genótipos Nanicão e FHIA-02 nos dois ciclos, para Bucaneiro e PA42-44, no primeiro ciclo, e para PV42-68 e ST12-31 no segundo ciclo de produção. Associações com relativa estabilidade foram apresentadas pelos genótipos Nanicão, Bucaneiro e PV42-142. Entretanto , Lima Neto et al. (2003), encontraram para esta correlação, estimativas positivas e significativas para quase todos os genótipos estudados.

Valores estatisticamente significativos para a associação peso do cacho e número de folhas vivas na colheita, foram encontrados para os genótipos Grande naine, Bucaneiro, PV42-68 e PV42-142 no segundo ciclo de produção e para o híbrido ST12-31 nos dois ciclos. A maioria dos genótipos apresentaram valores não-significativos e positivos para a associação nos dois ciclos de produção, o que confirma os resultados obtidos por outros autores (LIMA NETO et al., 2003; SIQUEIRA, 1984). Os genótipos FHIA-02, PA42-44 e Pacovan apresentaram estabilidade, enquanto nos demais as estimativas variaram muito. De acordo com o panorama geral apresentado pela correlação neste trabalho e noutros revisados, pode-se inferir que os caracteres não apresentam uma correlação expressiva para um programa de melhoramento genético.

As correlações entre peso do cacho e número de dias do plantio à colheita foram predominantemente negativas e não-significativas. Correlações significativas e negativas ocorreram em Grande naine, Bucaneiro e PA4244 (segundo ciclo), PV42-68, PV42-85 e PV42-142 (primeiro) e ST12-31 (nos dois ciclos). As estimativas oscilaram bastante entre os ciclos, sendo estáveis apenas nos genótipos Nanicão e ST12-31. A variedade Prata anã, neste trabalho e no de Lima Neto et al. (2003), apresentou estimativas não-significativas para essa correlação.

Para a associação entre peso do cacho e o período do florescimento a colheita, verifica-se que a maioria dos genótipos exibiu valores não significativos e positivos.

Ciênc. agrotec., Lavras, v. 30, n. 1, p. 21-30, jan./fev., 2006 
Os genótipos Grande naine, nos dois ciclos, Bucaneiro, PV42-85 e ST12-31 no segundo ciclo e PV42-142 no primeiro ciclo de produção apresentaram valores significativos para essa associação. As estimativas foram estáveis apenas para as variedades Grande naine, Pacovan e Prata anã.

$\mathrm{Na}$ Tabela 4, são apresentadas as correlações entre o peso do cacho e os caracteres observados na época da colheita, relativos ao peso da segunda penca, peso médio do fruto, comprimento externo e interno do fruto, diâmetro do fruto e espessura da casca, para cada genótipo de bananeira, nos dois ciclos de produção, e seus respectivos testes de significância.
As associações peso do cacho e peso da segunda penca foram estatisticamente significativas, positivas e com valores de alta magnitude na maioria dos genótipos estudados nos dois ciclos de produção. As correlações apresentaram estabilidade em Nanicão, Calipso, Prata anã, Pacovan, PV42-68 e PV42-85. Correlações negativas e não-significativas ocorreram nos híbridos PA42-44 e PV42-142 no segundo ciclo de produção e nãosignificativas nos dois ciclos em Calipso, PA42-44 e PV42-85. Os resultados obtidos permitem inferir que esta associação pode ser genética, concordando com Jaramillo (1982).

TABELA 4 - Correlações fenotípicas entre o peso do cacho e os caracteres avaliados na época da colheita (caracteres relacionados aos frutos) para cada genótipo, no primeiro e segundo ciclos de produção, com os respectivos testes de significância, Guanambi, BA, 2002 a 2003.

\begin{tabular}{|c|c|c|c|c|c|c|c|}
\hline \multirow[t]{2}{*}{ Genótipos } & \multicolumn{7}{|c|}{ Correlação entre o peso do cacho e os caracteres 1} \\
\hline & Ciclos & PSP & PMF & CEF & CIF & DMF & ECA \\
\hline \multirow[t]{2}{*}{ Grande naine } & $1^{\circ}$ & $0,75^{\mathrm{ns}}$ & $0,87^{*}$ & $0,82^{*}$ & $0,93^{*}$ & $0,84^{*}$ & $0,63^{\mathrm{ns}}$ \\
\hline & $2^{\circ}$ & $0,95^{*}$ & $0,84^{*}$ & $0,38^{\mathrm{ns}}$ & $0,71^{\mathrm{ns}}$ & $0,79^{*}$ & $0,85^{*}$ \\
\hline \multirow[t]{2}{*}{ Nanicão } & $1^{\circ}$ & $0,81^{*}$ & $0,89^{*}$ & $0,94^{*}$ & $0,98^{*}$ & $-0,16^{\mathrm{ns}}$ & $-0,59^{\mathrm{ns}}$ \\
\hline & $2^{\circ}$ & $0,94^{*}$ & $0,92^{*}$ & $0,77^{\mathrm{ns}}$ & $0,93^{*}$ & $0,72^{\text {ns }}$ & $0,59^{\mathrm{ns}}$ \\
\hline \multirow[t]{2}{*}{ FHIA-02 } & $1^{\circ}$ & $0,95^{*}$ & $0,87^{*}$ & $0,87^{*}$ & $0,96^{*}$ & $0,72^{\text {ns }}$ & $0,49^{\text {ns }}$ \\
\hline & $2^{\circ}$ & $0,51^{\mathrm{ns}}$ & $0,81^{*}$ & $0,27^{\mathrm{ns}}$ & $0,76^{\mathrm{ns}}$ & $0,81^{*}$ & $0,26^{\mathrm{ns}}$ \\
\hline \multirow[t]{2}{*}{ Bucaneiro } & $1^{\circ}$ & $0,89^{*}$ & $0,61^{\mathrm{ns}}$ & $0,54^{\mathrm{ns}}$ & $0,58^{\mathrm{ns}}$ & $0,63^{\mathrm{ns}}$ & $-0,32^{\mathrm{ns}}$ \\
\hline & $2^{\circ}$ & $0,66^{\mathrm{ns}}$ & $0,74^{\mathrm{ns}}$ & $0,85^{*}$ & $0,91^{*}$ & $0,96^{*}$ & $0,36^{\mathrm{ns}}$ \\
\hline \multirow[t]{2}{*}{ Calipso } & $1^{\mathrm{o}}$ & $0,75^{\mathrm{ns}}$ & $-0,44^{\mathrm{ns}}$ & $0,75^{\text {ns }}$ & $0,89^{*}$ & $0,83^{*}$ & $0,71^{\mathrm{ns}}$ \\
\hline & $2^{\circ}$ & $0,77^{\mathrm{ns}}$ & $0,91^{*}$ & $0,83^{*}$ & $0,92^{*}$ & $0,89^{*}$ & $-0,02^{\mathrm{ns}}$ \\
\hline \multirow[t]{2}{*}{ Ambrósia } & $1^{\circ}$ & $0,68^{\mathrm{ns}}$ & $0,97^{*}$ & $0,75^{\text {ns }}$ & $0,95^{*}$ & $0,94^{*}$ & $0,48^{\text {ns }}$ \\
\hline & $2^{\circ}$ & $0,88^{*}$ & $0,58^{\mathrm{ns}}$ & $0,83^{*}$ & $0,83^{*}$ & $0,04^{\mathrm{ns}}$ & $0,70^{\mathrm{ns}}$ \\
\hline \multirow{2}{*}{ Prata anã } & $1^{\circ}$ & $0,94^{*}$ & $0,91^{*}$ & $0,90^{*}$ & $0,86^{*}$ & $0,19^{\text {ns }}$ & $0,70^{\text {ns }}$ \\
\hline & $2^{\circ}$ & $0,90^{*}$ & $0,91^{*}$ & $0,88^{*}$ & $0,76^{\mathrm{ns}}$ & $0,45^{\mathrm{ns}}$ & $0,08^{\mathrm{ns}}$ \\
\hline \multirow[t]{2}{*}{ PA42-44 } & $1^{\circ}$ & $0,67^{\mathrm{ns}}$ & $-0,00^{\mathrm{ns}}$ & $-0,32^{\mathrm{ns}}$ & $-0,51^{\mathrm{ns}}$ & $-0,72^{\mathrm{ns}}$ & $0,20^{\mathrm{ns}}$ \\
\hline & $2^{\circ}$ & $-0,07^{\mathrm{ns}}$ & $-0,04^{\mathrm{ns}}$ & $-0,05^{\mathrm{ns}}$ & $-0,04^{\mathrm{ns}}$ & $0,04^{\mathrm{ns}}$ & $-0,53^{\text {ns }}$ \\
\hline \multirow{2}{*}{ Pacovan } & $1^{\mathrm{o}}$ & $0,90^{*}$ & $0,85^{*}$ & $0,79^{*}$ & $0,90^{*}$ & $0,59^{\text {ns }}$ & $-0,95^{*}$ \\
\hline & $2^{\circ}$ & $0,86^{*}$ & $0,53^{\mathrm{ns}}$ & $0,94^{*}$ & $0,75^{\text {ns }}$ & $0,61^{\mathrm{ns}}$ & $0,98^{*}$ \\
\hline \multirow[t]{2}{*}{ PV42-68 } & $1^{\circ}$ & $0,95^{*}$ & $0,38^{\text {ns }}$ & $0,70^{\mathrm{ns}}$ & $0,73^{\text {ns }}$ & $0,78^{*}$ & $0,41^{\mathrm{ns}}$ \\
\hline & $2^{\circ}$ & $0,95^{*}$ & $0,65^{\mathrm{ns}}$ & $0,33^{\mathrm{ns}}$ & $-0,46^{\mathrm{ns}}$ & $0,23^{\mathrm{ns}}$ & $-0,80^{*}$ \\
\hline \multirow[t]{2}{*}{ PV42-85 } & $1^{\circ}$ & $0,61^{\mathrm{ns}}$ & $0,73^{\mathrm{ns}}$ & $0,93^{*}$ & $0,80^{*}$ & $0,89^{*}$ & $0,89^{*}$ \\
\hline & $2^{\circ}$ & $0,55^{\mathrm{ns}}$ & $0,79^{*}$ & $-0,34^{\mathrm{ns}}$ & $0,51^{\mathrm{ns}}$ & $0,94^{*}$ & $-0,31^{\mathrm{ns}}$ \\
\hline \multirow[t]{2}{*}{ PV42-142 } & $1^{\circ}$ & $0,95^{*}$ & $0,98^{*}$ & $0,86^{*}$ & $0,83^{*}$ & $0,92^{*}$ & $0,25^{\mathrm{ns}}$ \\
\hline & $2^{\circ}$ & $-0,24^{\mathrm{ns}}$ & $0,60^{\mathrm{ns}}$ & $-0,11^{\mathrm{ns}}$ & $0,37^{\mathrm{ns}}$ & $0,43^{\mathrm{ns}}$ & $-0,55^{\mathrm{ns}}$ \\
\hline \multirow{2}{*}{ ST12-31 } & $1^{\mathrm{o}}$ & $0,66^{\mathrm{ns}}$ & $0,97^{*}$ & $0,88^{*}$ & $0,97^{*}$ & $0,88^{*}$ & $0,23^{\text {ns }}$ \\
\hline & $2^{\circ}$ & $0,87^{*}$ & $0,58^{\mathrm{ns}}$ & $0,60^{\mathrm{ns}}$ & $0,77^{\mathrm{ns}}$ & $0,60^{\mathrm{ns}}$ & $-0,22^{\mathrm{ns}}$ \\
\hline
\end{tabular}

1/PSP: Peso da segunda penca; PMF: Peso do fruto; CEF: Comprimento externo do fruto; CIF: Comprimento interno do fruto; DMF: Diâmetro do fruto; ECA: Espessura da casca.

*:Significativo ao nível de $5 \%$ de probabilidade; ns: não-significativo ao nível de $5 \%$ de probabilidade. 
A associação peso do cacho e peso médio dos frutos mostrou-se predominantemente significativa e positiva para a maioria dos genótipos nos dois ciclos de produção. Valores negativos e não-significativos para essa correlação foram observados para Calipso no primeiro ciclo e PA42-44 nos dois ciclos de produção. Os genótipos Grande naine, Nanicão, FHIA-02, Bucaneiro, Prata anã, PA42-44 e PV42-85 apresentaram estabilidade. Oscilação acentuada entre os ciclos para as estimativas foi expressa pelo híbrido Calipso. A tendência geral encontrada para esta correlação assemelhase a obtida por Lima Neto et al. (2003). Assim, pode-se inferir que os caracteres peso do cacho e peso médio dos frutos devem estar positivamente correlacionados.

As correlações peso do cacho e comprimento externo do fruto foram predominantemente significativas e positivas nos dois ciclos de produção, como em Lima Neto et al. (2003). Valores negativos e não-significativos foram apresentados pelos genótipos PA42-44 nos dois ciclos, PV42-85 e PV42142 no segundo ciclo. Estabilidade nos valores das correlações nos dois ciclos foram exibidas pelos genótipos Calipso, Ambrosia e Prata anã. A prevalência de valores positivos e significativos indica a existência de uma associação genética entre peso do cacho e comprimento do fruto.

Também a associação entre peso do cacho e comprimento interno do fruto apresentou, na maioria dos casos, valores estatisticamente significativos e positivos, denotando interação genética, embora valores negativos e não-significativos tenham sido encontrados nos genótipos PA42-44 nos dois ciclos e no PV42-68 no segundo ciclo. Estabilidade nos valores das correlações nos dois ciclos de produção foram verificadas em Nanicão, Calipso, Ambrosia e Prata anã e oscilações de grande magnitude em PV42-68 e PV42-142.

Para a associação peso do cacho e diâmetro do fruto, aproximadamente 50\% dos valores encontrados foram estatisticamente significativos e positivos. Correlações negativas e não significativas foram encontradas nos genótipos Nanicão e PA42-44 no primeiro ciclo de produção. Estabilidade entre os valores das estimativas no primeiro e no segundo ciclos foram apresentadas por Grande naine, FHIA-02, Calipso, Pacovan e PV42-85. Oscilações acentuadas ocorreram em Nanicão, Ambrosia, PA42-44, PV42-68 e PV42-142.

Analisando as correlações entre peso do cacho e espessura da casca, observa-se que foram predominantemente não-significativas para a maioria dos genótipos nos dois ciclos de produção. Verificaram-se intensas oscilações nos valores das estimativas entre os ciclos em Nanicão, Bucaneiro, Calipso, Prata anã, PA42-
44, Pacovan, PV42-68, PV42-85, PV42-142 e ST12-31. Valores estatisticamente significativos foram encontrados em Grande naine e PV42-68 no segundo ciclo, em PV42-85 no primeiro e Pacovan nos dois ciclos de produção.

As associações possíveis para os 21 caracteres considerando-se simultaneamente todos os genótipos ao longo dos dois ciclos, encontram-se na Tabela 5.

Aproximadamente $43 \%$ das associações entre todos os caracteres envolvendo simultaneamente os genótipos e ciclos foram significativas, e $57 \%$ nãosignificativas. Do total das associações observadas, $46 \%$ foram negativas e $54 \%$ positivas. Dentre as correlações estatisticamente significativas, destacam-se pela magnitude das estimativas: PCA x APL (-0,78); PRA x APL $(-0,78)$; PPE x APL (-0,77); DEN x APL $(-0,86)$; NPE x APL ($0,91)$; NFR x APL $(-0,82)$; DMF x APL $(-0,72)$; NFC x NFF $(0,93)$; NDC x NDF $(0,95)$; PFC x NDF $(-0,91)$; PSP X NDF $(0,80)$; PRA x PCA $(0,86)$; PPE x PCA $(0,99)$; DEN x PCA $(0,75)$; NPE x PCA $(0,83)$; NFR X PCA $(0,94)$; PFC x PCA ($0,74)$; PSP x PCA (0,92); PMF x PCA $(0,79)$; CEF x PCA $(0,78)$; ECA x PCA $(-0,81)$; PPE X PRA $(0,85)$; NPE x PRA $(0,75)$; NFR x PRA $(0,76)$; PSP x PRA $(0,75)$; PMF x PRA $(0,81)$; CEF x PRA $(0,78)$; DEN x PPE $(0,76)$; NPE x PPE $(0,83)$; NFR x PPE $(0,94)$; PFC x PPE $(-0,74)$; PSP x PPE $(0,93)$; PMF x PPE $(0,79)$; CEF x PPE $(0,78)$; ECA x PPE ($0,81)$; CIF x CEN $(0,72)$; NPE x DEN $(0,92)$; NFR x DEN $(0,90)$; ECA x DEN $(-0,74)$; NFR x NPE $(0,93)$; ECA x NPE $(-$ $0,70)$; PSP x NFR $(0,80)$; ECA x NFR $(-0,85)$; PFC x NDC ($0,75)$; PSP x PFC $(-0,89)$; CEF x PFC $(-0,84)$; ECA x PFC $(0,73)$; PMF x PSP $(0,81)$; CEF x PSP $(0,88)$; ECA x PSP (0,73); CEF x PMF $(0,88)$.

Analisando as correlações entre o caráter peso do cacho e os 20 demais caracteres, para todos os genótipos avaliados no decorrer dos ciclos (Tabela 5), verifica-se a prevalência de estimativas significativas e positivas. As associações entre o peso do cacho e perímetro do pseudocaule, número de folhas vivas no florescimento e na colheita, número de dias do plantio à colheita, comprimento do engaço, comprimento interno do fruto foram não significativas. Algumas correlações significativas foram negativas como entre peso do cacho e altura da planta, período do florescimento à colheita $\mathrm{e}$ espessura da casca. Dentre as estimativas significativas observadas, várias se destacaram pela magnitude como entre o peso do cacho e os caracteres altura da planta, período do florescimento à colheita, peso das pencas, diâmetro do engaço, número de pencas, número de frutos, peso da segunda penca, peso médio dos frutos, comprimento externo dos frutos e espessura da casca.

Ciênc. agrotec., Lavras, v. 30, n. 1, p. 21-30, jan./fev., 2006 
TABELA 5 - Correlações fenotípicas entre todos os caracteres observados, considerando-se simultaneamente todos os genótipos ao longo dos dois ciclos avaliados, com os respectivos testes de significância, Guanambi, BA, 2002 a 2003.

\begin{tabular}{|c|c|c|c|c|c|c|c|c|c|c|c|c|c|c|c|c|c|}
\hline$\overline{\mathbf{F F}}$ & NDF & PCA & PRA & PPE & CEN & DEN & NPE & NFR & NFC & NDC & PFC & PSP & PMF & CEF & CIF & DMF & ECA \\
\hline$\overline{14^{\mathrm{ns}}}$ & $-0,11^{\mathrm{ns}}$ & $-0,78^{*}$ & $-0,78^{*}$ & $-0,77^{*}$ & $0,33^{\mathrm{ns}}$ & $-0,86^{*}$ & $-0,91^{*}$ & $-0,82^{*}$ & $-0,38^{\mathrm{ns}}$ & $0,04^{\mathrm{ns}}$ & $0,29^{\text {ns }}$ & $-0,52^{*}$ & $-0,53^{*}$ & $-0,42^{\mathrm{ns}}$ & $0,12^{\mathrm{ns}}$ & $-0,77^{*}$ & $0,67^{*}$ \\
\hline $105^{\mathrm{ns}}$ & $0,54^{*}$ & $-0,07^{\mathrm{ns}}$ & $-0,40^{\mathrm{ns}}$ & $-0,05^{\mathrm{ns}}$ & $-0,52^{*}$ & $0,20^{\mathrm{ns}}$ & $-0,08^{\text {ns }}$ & $0,10^{\mathrm{ns}}$ & $-0,12^{\mathrm{ns}}$ & $0,67^{*}$ & $-0,28^{\mathrm{ns}}$ & $0,04^{\mathrm{ns}}$ & $-0,45^{\mathrm{ns}}$ & $-0,20^{\mathrm{ns}}$ & $-0,58^{*}$ & $-0,23^{\text {ns }}$ & $-0,16^{\mathrm{ns}}$ \\
\hline \multirow[t]{17}{*}{ - } & $-0,38^{\mathrm{ns}}$ & $-0,14^{\mathrm{ns}}$ & $-0,22^{\mathrm{ns}}$ & $-0,13^{\text {ns }}$ & $-0,44^{*}$ & $0,15^{\mathrm{ns}}$ & $0,26^{\mathrm{ns}}$ & $0,04^{\mathrm{ns}}$ & $0,93^{*}$ & $-0,25^{\mathrm{ns}}$ & $0,52^{*}$ & $-0,32^{\mathrm{ns}}$ & $-0,22^{\mathrm{ns}}$ & $-0,42^{\mathrm{ns}}$ & $-0,20^{\text {ns }}$ & $-0,03^{\text {ns }}$ & $0,14^{\mathrm{ns}}$ \\
\hline & - & $0,61^{*}$ & $0,29^{\mathrm{ns}}$ & $0,62^{*}$ & $-0,06^{\mathrm{ns}}$ & $0,33^{\mathrm{ns}}$ & $0,25^{\mathrm{ns}}$ & $0,58^{*}$ & $-0,34^{\mathrm{ns}}$ & $0,95^{*}$ & $-0,91^{*}$ & $0,80^{*}$ & $0,37^{\mathrm{ns}}$ & $0,62^{*}$ & $-0,06^{\mathrm{ns}}$ & $0,03^{\mathrm{ns}}$ & $-0,63^{*}$ \\
\hline & & - & $0,86^{*}$ & $0,99^{*}$ & $-0,11^{\text {ns }}$ & $0,75^{*}$ & $0,83^{*}$ & $0,94^{*}$ & $0,09^{\mathrm{ns}}$ & $0,44^{\mathrm{ns}}$ & $-0,74^{*}$ & $0,92^{*}$ & $0,79^{*}$ & $0,78^{*}$ & $0,08^{\mathrm{ns}}$ & $0,62^{*}$ & $-0,81^{*}$ \\
\hline & & & - & $0,85^{*}$ & $0,20^{\text {ns }}$ & $0,64^{*}$ & $0,75^{*}$ & $0,76^{*}$ & $0,03^{\text {ns }}$ & $0,05^{\mathrm{ns}}$ & $-0,57^{\mathrm{ns}}$ & $0,75^{*}$ & $0,81^{*}$ & $0,78^{*}$ & $0,28^{\mathrm{ns}}$ & $0,59^{*}$ & $-0,67^{*}$ \\
\hline & & & & $\begin{array}{ll}- & \text { r }\end{array}$ & $-0,12^{\mathrm{ns}}$ & $0,76^{*}$ & $0,83^{*}$ & $0,94^{*}$ & $0,09^{\mathrm{ns}}$ & $0,46^{\mathrm{ns}}$ & $-0,74^{*}$ & $0,93^{*}$ & $0,79^{*}$ & $0,78^{*}$ & $0,07^{\mathrm{ns}}$ & $0,62^{*}$ & $-0,81^{*}$ \\
\hline & & & & & - & $-0,51^{*}$ & $-0,38^{\text {ns }}$ & $-0,33^{\mathrm{ns}}$ & $-0,43^{\text {ns }}$ & $-0,19^{\text {ns }}$ & $-0,13^{\mathrm{ns}}$ & $0,02^{\text {ns }}$ & $0,29^{\mathrm{ns}}$ & $0,40^{\mathrm{ns}}$ & $0,72^{*}$ & $-0,43^{\text {ns }}$ & $0,11^{\mathrm{ns}}$ \\
\hline & & & & & & - & $0,92^{*}$ & $0,90^{*}$ & $0,34^{\mathrm{ns}}$ & $0,25^{\mathrm{ns}}$ & $-0,38^{\mathrm{ns}}$ & $0,52^{*}$ & $0,28^{\mathrm{ns}}$ & $0,27^{\mathrm{ns}}$ & $-0,41^{\mathrm{ns}}$ & $0,64^{*}$ & $-0,74^{*}$ \\
\hline & & & & & & & - & $0,93^{*}$ & $0,48^{*}$ & $0,15^{\mathrm{ns}}$ & $-0,33^{\mathrm{ns}}$ & $0,58^{*}$ & $0,49^{*}$ & $0,37^{\mathrm{ns}}$ & $-0,21^{\mathrm{ns}}$ & $0,69^{*}$ & $-0,70^{*}$ \\
\hline & & & & & & & & - & $0,24^{\mathrm{ns}}$ & $0,46^{\mathrm{ns}}$ & $-0,63^{*}$ & $0,80^{*}$ & $0,57^{*}$ & $0,56^{*}$ & $-0,17^{\text {ns }}$ & $0,61^{*}$ & $-0,85^{*}$ \\
\hline & & & & & & & & & - & $-0,27^{\text {ns }}$ & $-0,41^{\mathrm{ns}}$ & $-0,14^{\mathrm{ns}}$ & $-0,02^{\mathrm{ns}}$ & $-0,26^{\mathrm{ns}}$ & $-0,22^{\mathrm{ns}}$ & $0,18^{\mathrm{ns}}$ & $-0,04^{\text {ns }}$ \\
\hline & & & & & & & & & & - & $-0,75^{*}$ & $0,65^{*}$ & $0,18^{\mathrm{ns}}$ & $0,40^{\mathrm{ns}}$ & $-0,18^{\mathrm{ns}}$ & $-0,07^{\text {ns }}$ & $-0,49^{*}$ \\
\hline & & & & & & & & & & & - & $-0,89^{*}$ & $-0,57^{*}$ & $-0,84^{*}$ & $-0,11^{\mathrm{ns}}$ & $-0,15^{\text {ns }}$ & $0,73^{*}$ \\
\hline & & & & & & & & & & & & - & $0,81^{*}$ & $0,88^{*}$ & $0,21^{\mathrm{ns}}$ & $0,44^{\mathrm{ns}}$ & $-0,73^{*}$ \\
\hline & & & & & & & & & & & & & - & $0,88^{*}$ & $0,60^{*}$ & $0,46^{\mathrm{ns}}$ & $-0,43^{\text {ns }}$ \\
\hline & & & & & & & & & & & & & & - & $0,53^{*}$ & $0,20^{\mathrm{ns}}$ & $-0,62^{*}$ \\
\hline & & & & & & & & & & & & & & & $\begin{array}{ll}- & -1 \\
\end{array}$ & $-0,15^{\text {ns }}$ & $0,13^{\text {ns }}$ \\
\hline & & & & & & & & & & & & & & & & - & $-0,36^{\mathrm{ns}}$ \\
\hline
\end{tabular}

*:Significativo ao nível de $5 \%$ de probabilidade. ns: não-significativo ao nível de $5 \%$ de probabilidade.

\section{CONCLUSÕES}

As correlações entre o peso do cacho e os demais caracteres estudados, variaram entre genótipos e ciclos.

As associações entre o peso do cacho e os caracteres da planta, de forma geral foram nãosignificativas, e entre o peso do cacho e os caracteres do cacho significativas e positivas.

As correlações entre os caracteres envolvendo todos os genótipos nos dois ciclos foram predominantemente positivas e não-significativas, entretanto, as associações entre os caracteres do cacho foram em maioria significativas, positivas e com valores expressivos.

\section{REFERÊNCIAS BIBLIOGRÁFICAS}

CARVALHO, P. C. L. de. Estabelecimento de descritores botânicos-agronômicos para caracterização de banana (Musa spp). 1995. 190 f. Dissertação (Mestrado em Fruticultura Tropical) - Universidade Federal da Bahia, Cruz das Almas, 1995.

DONATO, S. L. R. Comportamento de variedades e híbridos de bananeira (Musa spp.), em primeiro ciclo de produção no Sudoeste da Bahia, Região de Guanambi. 2003. $115 \mathrm{f}$. Dissertação (Mestrado em Ciência e Tecnologia de
Sementes) - Faculdade de Agronomia "Eliseu Maciel", Universidade Federal de Pelotas, Pelotas, 2003.

FERNANDEZ-CALDAS, E.; GARCIA, V.; PEREZ-GARCIA, V.; DIAZ, A. Análisis foliar del plátano en dos fases de su desarrollo: floración y corte. Fruits, Paris, v. 32, n. 11, p. 665-671, nov. 1977.

FLORES, J. C. de O. Avaliação de cultivares e híbridos de bananeira (Musa spp.) em quatro ciclos de produção em cruz das Almas, BA. 2000. 109 f. Dissertação (Mestrado em Fruticultura Tropical) - Universidade Federal da Bahia, Cruz das Almas, 2000.

GARCIA, V.; FERNANDEZ-CALDAS, E.; DIAZ, A.; BRAVO, J. J. Análisis foliar del plátano en dos fases de su floración. Fruits, Paris, v. 32, n. 9, p. 525-534, set. 1977.

GOMES, F. P. O uso da regressão na análise de variância. In: _ Curso de estatística experimental. 11. ed. Piracicaba: Nobel, 1985.p. 227-251.

HASSELO, H. N. An evaluation of the circumference of the pseudostem as a growth index for the Gros Michel banana. Tropical Agriculture, Londres, v. 39, n. 1, p. 57-63, Jan. 1962. 
HOLDER, G. D.; CUMBS, F. A. Effects of water supply during floral initiation and differentiation on female flower production by Robusta banana. Experimental Agriculture, New York, v. 18, n. 2, p. 183-193, 1982.

IUCHI, V. L.; RODRIGUES, J. A. S.; MANICA, I.; OLIVEIRA, L. M. Parcelamento do adubo nitrogenado e potássico em bananeira (Musa sp.) cv. 'Prata'. In: CONGRESSO BRASILEIRO DE FRUTICULTURA, 5., 1979, Pelotas. Anais... Pelotas: Sociedade Brasileira de Fruticultura, 1979. v. 1, p. 109-117.

JARAMILLO, R. C. Las principales características morfológicas del fruto de banano, variedade Cavendish Gigante (Musa AAA) em Costa Rica. Costa Rica: Upeb, 1982. 42 p.

LIMA NETO, F. P.; SILVA, S. de O. e; FLORES, J. C. O.; JESUS, O. N.; PAIVA, L. E. Relação entre caracteres de rendimento e desenvolvimento em genótipos de bananeira. Magistra, Cruz das Almas, v. 15, n. 2, p. 275-281, 2003.

LOSSOIS, P. Recherche d'une méthode de prévision des récoltes en culture bananière. Fruits, Paris, v. 18, n. 6, p. 283-293, jan. 1963.

PÁDUA, T. Caracterização agronômica do cacho da bananeira 'Prata'. 1978. 117 f. Dissertação (Mestrado) Escola Superior de Agricultura de Lavras, Lavras, 1978.
RIBEIRO JÚNIOR, J. I. Análises estatísticas no SAEG. Viçosa: UFV, 2001. $301 \mathrm{p}$.

SILVA, S. de O. e; ALVES, E. J.; LIMA, M. B.; SILVEIRA J. R. da S. Bananeira. In: BRUCKNER, C. H. (Ed.). Melhoramento de fruteiras tropicais. Viçosa: UFV, 2002. p. 101-157.

SILVA, S. de O. e; ROCHA, S. A.; ALVES, E. J.; CREDICO, M. de; PASSOS, A. R. Caracterização morfológica e avaliação de cultivares e híbridos de bananeira. Revista Brasileira de Fruticultura, Jaboticabal, v. 22, n. 2, p. 161169, ago. 2000.

SIQUEIRA, D. L. Variabilidade e correlações de caracteres em clones da bananeira 'Prata'. 1984. 66 f. Dissertação (Mestrado) - Escola Superior de Agricultura de Lavras, Lavras, 1984.

VARGAS, G. Comparación em calidades y rendimientos de los clones "Gran Enano" y "Valery". Tesis em preparación (Doutorado) - Faculdad de Agronomia, Universidad de Costa Rica, San José, 1983.

VENCOVSKY, R.; BARRIGA, P. Associação entre caracteres. In: Genética biométrica no

fitomelhoramento. Ribeirão Preto: Sociedade Brasileira de Genética, 1992. p. 335-434.

Ciênc. agrotec., Lavras, v. 30, n. 1, p. 21-30, jan./fev., 2006 